\title{
The Determinants of the Time to Efficiency in Options Markets : A Survival Analysis Approach
}

\author{
LAURENT DEVILLE* \\ FABRICE RIVA $^{\dagger}$ \\ October 2004 \\ First version. Comments welcome
}

\begin{abstract}
This paper examines the determinants of the time it takes for an index options market to be brought back to efficiency after put-call parity deviations, using intraday transactions data from the French CAC 40 index options over the August 2000 - July 2001 period. We address this issue through survival analysis which allows us to characterize how differences in market conditions influence the expected time before the market reaches the no-arbitrage condition. We find that moneyness, maturity, trading volume as well as trade imbalances in call and put options, and volatility are important in understanding why some arbitrage opportunities disappear faster than others. After controlling for differences in the trading environnement, we find evidence of a negative relationship between the existence of ETFs on the index and the time to efficiency.
\end{abstract}

KeY Words: Index Options, Market efficiency, Survival Analysis, Exchange Traded Funds.

JEL Classification: C41, G13, G14.

*CNRS; CEREG (UMR 7088); Paris Dauphine University; Place du Maréchal de Lattre de Tassigny; 75775 Paris Cedex 16, France; Tel.: +33 (0)1 440545 36; Fax: +33 (0)1 440540 23; e-mail: Laurent.Deville@dauphine.fr

${ }^{\dagger}$ CEREG (UMR 7088); Paris Dauphine University; Place du Maréchal de Lattre de Tassigny; 75775 Paris Cedex 16, France; Tel.: +33 (0)1 440549 88; Fax: +33 (0)1 440540 23; e-mail: Fabrice.Riva@ dauphine.fr 


\section{Introduction}

Research on options markets efficiency based on arbitrage relationships commonly agree that these relationships hold on average. However, whether it be on $\mathrm{US}^{1}$ or European markets ${ }^{2}$, frequent and significant distortions have been evidenced, whatever the quality of the data, the kind of underlying asset and how carefully the computations of profits are led. The most striking evidence is provided by Kamara and Miller (1995) on the S\&P 500 options contract from May 1986 through May 1989. They find evidence of frequent put-call parity violations, even with a European contract, prices matched within a minute and accounting for transaction costs and dividends. Sometimes, options markets thus appear to be incompatible with no arbitrage prices and therefore with efficiency.

However, as pointed out in Chordia, Roll and Subrahmanyam (2004), "efficiency does not just congeal from spontaneous combustion" but is a process that depends on individual actions and, as a result, takes time. Efficient is the market where, after a distortion has been identified, prices revert rapidly enough to stop subsequent arbitrage trades. Efficiency will depend on the traders' ability to realize riskless abnormal profits given the information that the market prices are not compatible with no-arbitrage at a given time. On options markets, two principal ways have been followed since now to assess this kind of efficiency: one based on the computations of accessible ex ante profits and the other based on the identification of the determinants of the immediate (ex post) profits.

Transactions cannot be immediate after an opportunity has been identified. The prices a trader can get may differ from the ones he observed. The index level may move and the market makers could have adjusted their quotes both to account for this variation and, if the case need be, to protect themselves from arbitrage trades. Thus, some efficiency tests are based on the level of ex ante rather than ex post profits. The ex ante profits are the profits an arbitrageur can earn when being imposed an arbitrary "no trade" period. Kamara and Miller (1995), for the S\&P 500 index options, Mittnik and Rieken (2000) for the German DAX 30 index options and Deville (2004) for the French CAC 40 index options show that ex ante profits decrease with the

\footnotetext{
${ }^{1}$ Put-call parity empirical studies include Gould and Galai (1974) on OTC options, Klemkoski and Resnick (1979, 1980) on stock options traded on the CBOE, Evnine and Rudd (1985), Chance (1987), Finucane (1991) and Wagner, Ellis and Dubofsky (1996) on S\&P 100, and Kamara and Miller (1995), Ackert and Tian (2001) and Bharadwaj and Wiggins (2001) on S\&P 500. The latter two studies test other arbitrage relationships, such as the box-spread, also tested by Billingsley and Chance (1985), Chance (1987) and Ackert and Tian (1998).

${ }^{2}$ For empirical tests of arbitrage relationships on European index contracts, see Puttonen (1993) for the Finnish market, Chesney, Gibson and Loubergé (1995) for the Swiss market, Cavallo and Mammola (2000) and Cassese and Guidolin (2001) for the Italian market, and Capelle-Blancard and Chaudhury (2001) and Deville (2004) for the French market.
} 
length of the no-trade window. Hence, market prices adjust after a deviation occurred but not instantaneously and arbitrage opportunities persist for a while. Despite the introduction of a time-dimension in efficiency tests, ex ante tests still focus on the level of profits and are by no means truly dynamical although efficiency is.

To explain why apparent profits may appear on index options markets, Kamara and Miller (1995) regress the level of ex post put-call parity arbitrage profits on explanatory variables with their daily data set of S\&P 500 index options closing prices. Using a bootstrapped Tobit regression which allows them to appropriately handle the censored observations that are compatible with no-arbitrage, they show that the profit can be explained by liquidity risk factors. Hence, rather than reflecting inefficiency, ex post profits appear to be premia for the liquidity risk faced by investors who may engage in arbitrage trades. Following the same methodology, Ackert and Tian (2001) roughly find the same results for the various arbitrage relationships they test for the S\&P 500 index options.

Existing studies agree on the fact that profits tend to decrease as time goes by and that liquidity is an important determinant of the size of the violations. However, they do not explain why and how prices go back to efficiency levels. Our study precisely aims at understanding the process by which prices revert to levels compatible with no-arbitrage. In a previous paper, Deville (2004) measures the options market (in)efficiency as the time prices remain incompatible with the no-arbitrage levels implied by the put-call parity relationship. This measure is truly dynamic and uses the whole information set available with intraday data. Every index level and transaction price subsequent to the identification of a distortion is actually considered. Since then, this measure should prove most appropriate in identifying the determinants of efficiency or, in other words, in studying how efficiency emerges in the options markets.

Since we work with durations rather than profits, survival analysis naturally appears to be the most suitable tool to extract the factors affecting market efficiency. This econometric tool, that is common in biostatistics but rarely used in financial research, is based on the probability of an observation of dying in the next instant, provided that it has lived since then. Our population is the set of matched pairs of call and put transactions that are not compatible with put-call parity. We consider an observation as 'alive' as long as the profit resulting from the construction of the arbitrage portfolio remains positive. It is considered as 'dead' as soon as the built portfolio leads to a negative or zero profit. Furthermore, this approach is the most appropriate method to accommodate an important feature of time to efficiency: censored observations i.e. matched pairs that still exhibit a positive profit prior to the market close.

We use this methodology for the French CAC 40 index options from August 1, 2000 through July 31,2001 . This period surrounds January 21, 2001, the launching date of the ETF that 
replicates the CAC 40 index. Survival analysis allows us to compute the probability that an arbitrage opportunity dies conditionally to the fact that is has lived since. One interesting feature of the this probability is that it decreases with time: the more a deviation lasts, the more it has chance to last one more instant. Hence, a deviation that is not quickly brought back to efficiency may last for a long time, and this may illustrate the fact that some opportunities are exploited by arbitrageurs while some other are not worth the trouble. We find that the time to efficiency is negatively linked with several explanatory variables representing the activity on the options market, the volatility on the underlying asset and the possibility to trade the CAC 40 index through ETFs. We also document that differences in option characteristics lead to significant changes in the probability for an arbitrage opportunity to survive over a given time-interval.

The remainder of this paper is organized as follows. Section 2 explains the time to efficiency computations and reviews the survival analysis methodology. Section 3 describes the data. Section 4 presents the empirical results of our analysis of the determinants of the time to efficiency. Section 5 concludes.

\section{General methodology}

In our analysis, prices are considered efficient as soon as they are compatible with the put-call parity relationship. If it restricts our analysis to times when a pair of put and call transactions on the same serie are identified, this definition makes it possible to identify the forces driving the prices back to efficiency without resorting to any option pricing model. We briefly present the well-known put-call parity relationship and our a measure of efficiency based on the time it takes for a distortion to disappear, the time to efficiency. Then, we review the econometric tool we employ to isolates variables that modify this time, the survival analysis.

\subsection{Put-call parity relationship and arbitrage profits}

The notation used in the discussion is as follows:

$C_{t}$, European call premium at time $t$,

$P_{t}$, European put premium,

$K$, strike price,

$I_{t}$, index value,

$\tau$, time to maturity,

$r$, risk-free interest rate, 
$D$, present value of dividends paid from the transaction date until expiration, expressed in index points.

Under no-arbitrage, whenever put and call options having the same characteristics exist, their premiums must satisfy the put-call parity relationship ${ }^{3}$ (PCP):

$$
C_{t}-P_{t}=I_{t}-K e^{-r \tau}
$$

If equation (1) does not hold, the call option is under- or over-valued with respect to the put option and an arbitrage portfolio might be built by taking opposite positions in the "real" and the synthetic call. These strategies are called "long hedge" and "short hedge", dependent on the position that is held on the underlying asset. If dividends are to be paid during the life of the options, the initial positive flow generated by these two strategies, denoted $\pi_{L H}$ and $\pi_{S H}$ respectively, are equal to:

$$
\pi_{L H}=C_{t}-P_{t}-I_{t}+D+K e^{-r \tau}
$$

and:

$$
\pi_{S H}=P_{t}-C_{t}+I_{t}-D-K e^{-r \tau}
$$

These initial flows, calculated on the basis of transaction prices recorded at the pairing time, represent the ex post arbitrage profit. Both portfolios are to be held until expiration, at which time in-the-money options will be exercised and the index position cleared, leading to a zero terminal payoff. The measure of market efficiency we use in the remainder of the paper relies on the duration of the market inefficiencies rather than on the profit that could be earned from their exploitation.

\subsection{TTE as a measure of market (in)efficiency}

The measure of informational efficiency of derivatives markets we use in this paper, namely the time to efficiency (hereafter TTE), has been developed in Deville (2004). It consists in measuring how long it takes for the market prices to be brought back to no-arbitrage values, once a deviation has been identified.

The way TTE is computed is the following. We first match pairs of synchronous transactions of calls and puts having the same characteristics. We compute the initial (ex post) arbitrage profit using equations (2) and (3) as a function of the prices $P_{t}, C_{t}$ and $I_{t}$ which prevail at the

\footnotetext{
${ }^{3}$ Put-call parity was initially formalized by Stoll (1969) for at-the-money options and extended for non-payout protected options on dividend paying shares by Merton (1973).
} 
pairing time, $t$. If $\pi_{L H}\left(\pi_{S H}\right)$ is positive, the pair is classified as a long hedge (short hedge) deviation to put-call parity ${ }^{4}$. Then, each time a new market value is recorded for of one of the components of the arbitrage portfolio (put, call and index), its price is updated ${ }^{5}$. The profit resulting from the construction of the arbitrage portfolio at this time is then computed with this new set of prices using equations (2) and (3) for the long hedge and short hedge deviations, respectively. The updating process stops as soon as the profit value becomes zero or negative, since prices are then compatible with no arbitrage. The time to efficiency is the time it takes for the arbitrage profit to nullify, if ever.

As an example, consider a deviation occurring at time $t$ with $\pi_{L H}>0$. To compute the TTE, we look for the first subsequent modification of the value of either the put, the call or the index. Denote $t+1$ the time when this first modification occurs. If the only modification recorded at time $t+1$ is a variation in the index value, profit is computed at time $t+1$ with the set of prices $P_{t}, C_{t}$ and $I_{t+1}$ as:

$$
C_{t}-P_{t}-I_{t+1}+D+K e^{-r \tau}
$$

A negative or null value stops the computations and the time to efficiency equals the time elapsed between $t$ and $t+1$. In the case of a positive value, we look for the next modification in the value of one of the instruments considered. Denote $t+2$ the time when this second modification occurs. If the modifications recorded between $t$ and $t+1$ are a variation in the index value and in the call price, profit is computed at time $t+2$ with the set of prices $P_{t}, C_{t+2}$ and $I_{t+2}$ as:

$$
C_{t+2}-P_{t}-I_{t+2}+D+K e^{-r \tau}
$$

The process goes on in the case of a positive value and stops in the case of negative or null value, the time to efficiency being equal to the time elapsed between $t$ and $t+2$.

It is important to note that the time elapsed between two events is not a constant and depends on the frequency of dissemination of the index values and the transaction times of calls and puts of the same series. Another important feature of the TTE is that its computation relaxes

\footnotetext{
${ }^{4}$ Without transaction costs, $\pi_{L H}=-\pi_{S H}$ and every match deviates from put-call parity, either on the long hedge side or on the short hedge side. Transaction costs create a bandwidth within which prices can fluctuate without inducing any profitable arbitrage opportunity. They are not accounted for in this study since, as it can be seen in Deville (2004), distributions of TTEs are similar with and without transaction costs. It simply lowers the number of available data.

${ }^{5}$ It happens that multiple transactions of the same options are recorded in the same minute, with different transaction prices. In this case, we choose to keep the premium that leads to the smallest profit. In the case of long hedges (short hedges), we therefore use the most (less) expensive calls and the less (most) expensive puts. As a result, we obtain a lower limit on the time to efficiency. We have also estimated a higher limit deriving the profit with the premium that leads to the highest value. Results, available on demand, are only marginally modified since transactions, when recorded simultaneously, rarely present significantly different premiums.
} 
the synchronism constraint initially imposed on the prices of the instruments included in the arbitrage portfolio. The corresponding implicit hypothesis is that there is no price staleness. However, it is also the case for the classical ex ante tests of options markets efficiency in which the prices considered for the computation are the first one observed for each instrument once the execution delay has passed, without any constraint on synchronism.

The set of transactions and index values recorded before the market close do not necessarily induce a return to efficient prices. After the close, no information regarding the index value or options prices is disseminated until the market opening on the following day. In case of opening prices compatible with no arbitrage, we wouldn't be able to tell when the market became arbitrage-free again, whether it be just after the close or during the night. Rather than using opening prices to go on with TTE computation procedure, we stop it at the close. If the profit remains always positive throughout the remain of the trading day, no TTE is derived and we compute the time to close. The information we retain is that prices were still inefficient after this duration. Hence, the distribution of TTEs is right-censored. However, this doesn't mean that we have to drop such observations out of our sample since censorship can be handled with survival analysis.

\subsection{A brief review of survival analysis}

In this section, we develop an econometric model which aims at explaining the determinants of the time it takes for an arbitrage opportunity to disappear. We employ a statistical tool called survival analysis, a commonly used technique in the area of biostatistics, but whose applications in financial research are sparse $e^{6}$. Survival analysis is particularly well-suited in our context since we want to estimate the impact of market conditions for the survival of possibly censored times-to-efficiency.

\subsubsection{Basic quantities}

The most important quantity to describe time-to-event data is the survival function, which gives the probability of an individual (in our case, an arbitrage opportunity) surviving beyond time $t$. It is defined as:

$$
S(t)=\operatorname{Pr}(T>t)
$$

\footnotetext{
${ }^{6}$ A notable exception is the study of Lo, MacKinlay and Zhang (2002) in which survival analysis is used to model the time-to-execution of limit orders that liquidity suppliers post into the order book. Applications in the field of economics can be found in Greene (2002) and Kiefer (1988).
} 
When $T$ is a continuous random variable, the survival function is given by:

$$
S(t)=\int_{t}^{\infty} f(x) d x=1-F(t)
$$

where $f(\cdot)$ denotes the probability density function of $T, F(\cdot)$ its cumulated density function, and thus

$$
f(t)=-\frac{d S(t)}{d t}
$$

A quantity that is closely related to the survival function is the hazard function, which gives the probability that an event that has lasted up to time $t$ will terminate in the interval $[t, t+\Delta t]$. Its formal definition is given by:

$$
h(t)=\lim _{\Delta t \rightarrow 0} \frac{\operatorname{Pr}(t \leq T<t+\Delta t \mid T \geq t)}{\Delta t}
$$

and for continuous variables,

$$
h(t)=\frac{f(t)}{S(t)}=-\frac{d \log [S(t)]}{d t}
$$

Integrating the hazard function over $[0, t]$ yields the integrated or cumulated hazard function

$$
\Lambda(t)=\int_{0}^{t} h(x) d x=-\log [S(t)]
$$

whose main usage arises when one has to perform graphical checks of model adequacy (see section 4.3).

\subsubsection{Handling (right-) censored observations}

A particular feature in survival analysis is censoring. In our case, we are more particularly concerned with a given type of censoring called right censoring, which refers to situations where only lower bounds on lifetime are available for some trials.

We decided to treat as (right-)censored observations the arbitrage positions which are still profitable when the market closes $(5: 30 \mathrm{pm})$. Motivation to do so arises from the impossibility to compute the actual TTE for such positions as their lifetime includes at least one overnight 
non-trading period ${ }^{7}$.

The most common way to handle censored observations is based on the following approach. Uncensored observations provide information on the probability that an arbitrage opportunity has survived to its associated time to efficiency, which is approximately equal to the density function of $T$ at that time (denoted $T_{i}$ ). For right-censored observations, the appropriate quantity is the survival function as the only thing we know about the true time is that it is greater than the censoring time (denoted $C_{i}$ ). Putting things differently, and denoting $\delta_{i}=1_{\left\{T_{i} \leq C_{i}\right\}}, t_{i}=$ $\min \left(T_{i}, C_{i}\right)$ is observed when $\delta_{i}=1$ (no censoring) whereas the case $\delta_{i}=0$ corresponds to situations in which we know only that $T_{i}>C_{i}$ (censoring).

Under the assumption that the censoring time $C_{i}$ is independent from the true time $T_{i}$, we obtain the likelihood function as ${ }^{8}$ :

$$
\mathcal{L}=\prod_{i=1}^{n} f\left(t_{i}\right)^{\delta_{i}} S\left(t_{i}\right)^{\left(1-\delta_{i}\right)}
$$

\subsubsection{The Weibull distribution}

So far, we have not placed any structure on the distribution $T$ is drawn from. Among the different choices, we decided to focus on the Weibull distribution as it is both fairly general and mathematically tractable.

The Weibull distribution is a two-parameter distribution whose p.d.f. is 9 :

$$
f_{W}(t)=\alpha \lambda t^{\alpha-1} \exp \left(-\lambda t^{\alpha}\right), t>0
$$

and the associated survival function is:

$$
S_{W}(t)=\exp \left(-\lambda t^{\alpha}\right)
$$

From the definition of the hazard function in the continuous case, the hazard function when $T$ follows a Weibull distribution comes to be:

$$
h_{W}(t)=\alpha \lambda t^{\alpha-1}
$$

\footnotetext{
${ }^{7}$ As an alternative procedure, we could have discarded such arbitrage opportunities. Proceeding this way would bias the results on the true survival time as censored observation convey valuable information, namely the fact that the arbitrage opportunity has survived till its censoring time.

${ }^{8}$ Differently stated in our case, time-to-censoring (i.e. time to close) provides no information about what would have been the true time to efficiency.

${ }^{9} \alpha>0$ is a shape parameter whereas $\lambda>0$ is a scale parameter.
} 
so that the Weibull distribution can accommodate increasing $(\alpha>1)$, constant $(\alpha=1)$ or decreasing $(\alpha<1)$ hazard.

\subsubsection{Including covariates}

The general case The most common way to include covariates (explanatory variables) assumes a linear relationship between the log of survival time and the covariate values, namely:

$$
Y=\ln (T)=\mu+\gamma^{\prime} Z+\sigma U
$$

where $\gamma^{\prime}=\left(\gamma_{1}, \ldots, \gamma_{p}\right)$ is a vector of regression coefficients and $U$ is the error distribution. Such an approach is called accelerated failure time (AFT). To see this, let $S_{0}(t)$ denote the survival function of $T=e^{Y}$ when $Z$ is 0 , that is $S_{0}(t)$ is the survival function of $\exp (\mu+\sigma U)$. Then it comes :

$$
\begin{aligned}
\operatorname{Pr}[T>t \mid Z] & =\operatorname{Pr}[Y>\ln t \mid Z] \\
& =\operatorname{Pr}\left[\mu+\sigma U>\ln t-\gamma^{\prime} Z \mid Z\right] \\
& =\operatorname{Pr}\left[\exp (\mu+\sigma U)>t \exp \left(-\gamma^{\prime} Z\right) \mid Z\right] \\
& =S_{0}\left[t \exp \left(\gamma^{\prime} Z\right)\right]
\end{aligned}
$$

so that, the survival function being strictly decreasing, the effect of the explanatory variables in the original time scale is to accelerate (decelerate) time by a factor $\exp \left(-\gamma^{\prime} Z\right)$ when $\gamma$ is negative (positive).

The Weibull case Computations for the p.d.f of $Y=\ln T$ when $T$ follows a Weibull distribution yield:

$$
f_{Y}(y)=\alpha \lambda \exp \left(\alpha t-\lambda e^{\alpha t}\right),-\infty<y<+\infty
$$

Setting

$$
\left\{\begin{array}{l}
\lambda=\exp (-\mu / \sigma) \\
\alpha=1 / \sigma
\end{array}\right.
$$

$f_{Y}(y)$ is found to be:

$$
f_{Y}(y)=\frac{1}{\sigma} \exp \left(\frac{t-\mu}{\sigma}-\exp \left(\frac{t-\mu}{\sigma}\right)\right),-\infty<y<+\infty
$$


with an associated survival function:

$$
S_{Y}(y)=\exp (-\exp [(y-\mu) / \sigma])
$$

From (18), it comes that the p.d.f of the error term $U=(Y-\mu) / \sigma$ in the Weibull case is given by:

$$
f_{U}(u)=\exp \left(u-e^{u}\right)
$$

which is the p.d.f. of the standard extreme value distribution. Direct computations yield the corresponding survival function:

$$
S_{U}(u)=\exp (-\exp (u))
$$

Using (10), the likelihood function for right-censored data when $T$ follows a Weibull is given by:

$$
\begin{aligned}
\mathcal{L} & =\prod_{i=1}^{n}\left[f_{Y}(y)\right]^{\delta_{i}}\left[S_{Y}(y)\right]^{\delta_{i}} \\
& =\prod_{i=1}^{n}\left[\frac{1}{\sigma} f_{U}\left(\frac{y_{i}-\mu}{\sigma}\right)\right]^{\delta_{i}}\left[S_{U}\left(\frac{y_{i}-\mu}{\sigma}\right)\right]^{\delta_{i}}
\end{aligned}
$$

where $f_{Y}(y), S_{Y}(y), f_{U}(u)$ and $S_{U}(u)$ are given in (18) $-(21)$. Setting $\mu^{\prime}=\mu+\gamma^{\prime} Z$ and replacing $\mu$ by $\mu^{\prime}$ in (22) gives the likelihood function including covariates. This function will form the basis of our estimation procedure.

\section{The data}

We empirically investigate the determinants of the TTE for the CAC 40 index options contract for the 12 months period from August 2000 to July 2001, period surrounding the January 21, 2001, introduction of the ETF tracking the CAC 40 index on NextTrack. The CAC 40 index derivative contracts is the most traded contracts on the Marché des Options Négociables de Paris (MONEP), the French market for equity and index derivatives. In year 2000, the CAC 40 index option contract (PXL ticker) accounts for one third of the total open interest and one half of the number of contracts traded on the options market. The efficiency of this contract constitutes a benchmark of the MONEP efficiency. We present the specifications of options and ETF contracts on CAC 40 index and our data set of TTEs. 


\subsection{CAC 40 index options and ETFs contracts ${ }^{10}$}

The CAC 40 index consists of 40 stocks selected from the most active and representative of the various economic sectors quoted on Paris "Premier Marché". Its value is calculated continuously as the market value weighted average of the 40 stock prices, and is disseminated every 30 seconds by Euronext Paris. The index is managed by an independent committee, the "Conseil Scientifique des Indices", which adapts the index to reflect changes in the market or in the market value of the constituent stocks.

The CAC 40 index option is the most active contract of the MONEP with an average monthly volume of more than 7 millions traded contracts and of 1 billion Euros premium between August 2000 and July 2001. In contrast to the French stock market, which is order-driven, the MONEP is quote-driven with market-makers continuously competing for the order flow. Market makers have an obligation to maintain a permanent bid-ask spread for option series near the money and must publicly reply to any investor's price demand. Transactions on the MONEP are carried out by matching buy and sell orders. Orders offering the best execution conditions are given priority, with priority for orders at the same price determined by their time-stamp in the central order book. As it is the case for stock trading on Euronext Paris, after a fixing, the quote is continuously ensured from 9:02 to 17:30 on the automated system NSC until the closing fixing at $17: 35^{11}$.

The size of PXL contracts is equal to the value of the CAC 40 index multiplied by one Euro and the tick size is 0.1 index point. This contract is cash-settled ${ }^{12}$ and exclusively of European-style exercise. Trading covers eight rolling open maturities: three spot months, three quarterly maturities and two half-yearly maturities. The same expiration months are opened for the futures contract on the CAC 40 index, also traded on the MONEP. Strike prices are set at standard intervals of 50, 100 or 200 points depending on the expiration date. The series opened to trading are not necessarily the same for calls and puts. At every moment in time, at least three strike prices are listed: one "at the money" and two "out of the money". New series are created depending on the price changes of the CAC 40 index.

CAC 40 Master Unit, the first ETF quoted in Paris since January 22, 2001, is aimed at replicating price and performance of the CAC 40 index. Its initial value was $1 / 100^{\text {th }}$ of the

\footnotetext{
${ }^{10}$ Informations given in this subsection concerns the sample period and may have change since. More details on the market and the contracts specifications can be found in Deville (2004).

${ }^{11}$ On 23 April 2001, Euronext implemented a common market model in its three constituent market places, Paris, Amsterdam and Brussels. The continuous trading period ranges now from 9:00 for the open to 17:25 for the close, followed by a closing call auction (fixing) at 17:30.

${ }^{12}$ The settlement value is equal to the mean of all index values calculated and disseminated between 15:40 and 16:00 on the expiration day.
} 
index value. An annual dividend is paid, equal to the total amount of dividends accumulated by the fund minus management expenses of $0.30 \%$. ETFs are traded on two parallel markets, each governed by its own rules. The primary market is the issuing market, where the creation and redemption of parts of the fund can be carried out. Meanwhile, ETFs listed on Euronext can be traded on its secondary market, NextTrack.

As an open-ended fund, the assets under management can evolve over time, through the creation and redemption of trackers, which are the traded units of the fund. For example, the capital managed by the CAC 40 Master Unit issuer, namely Lyxor Asset Management, was 765,655 thousand Euros (corresponding to $17,338,211$ shares) after one year of quotation and 1, 038, 333 thousand Euros (34,750, 111 shares) after two years. NextTrack, the secondary market for the CAC 40 Master Unit, is almost organized as Euronext Paris Premier Marché. ETFs are traded continuously through an electronic order book accessible to both issuers and investors from 9:05 to 17:25. A closing auction is organized at 17:35 and trades after the Trading Hours can occur at prices within a price range of $1 \%$ around the the last disseminated indicative NAV. One difference from the usual French stock market trading is the obligatory presence of two committed market participants that provide liquidity by posting continuous quotes in the order book for a minimum order size. They have to maintain a maximum spread of $0.40 \%$ up to five millions Euros for the CAC 40 Master Unit.

\subsection{Data and TTE results}

The intraday data of transactions on the PXL contract have been extracted from the Euronext Paris Market Database from August 2000 to July 2001. For the 78,887 call and 92, 059 put transactions recorded, this database reports the strike price and the expiration month, and timestamped informations such as the premium and the number of contracts traded. Dividends delivered by the underlying asset must be accounted for, unless calls appear arbitrarily overvalued. French stocks usually deliver annual dividends in May or June. Discrete dividends have been extracted from Thomson Financial Datastream and expressed in term of CAC 40 index points on a daily basis. For each match, the present value of the dividends delivered between the trade and the expiration date are calculated using Euribor as a proxy for the risk-free interest rate. One week to one year Euribor rates have been obtained from Thomson Financial Datastream. The interest rate used for each matching is a linear interpolation of Euribor rates surrounding the time to maturity.

A matching pair is selected each time we observe a call and a put having the same exact characteristics (strike price and expiration month) traded within a one minute trade-interval. 
Each pairing is associated with the index value prevailing at the same time ${ }^{13}$, extracted from Euronext Database. Thus, we avoid asynchronous bias that can lead to an overestimation of market efficiency. Options with less than two days and more than one year to expiration as well as trades recorded with a premium less than 2 Euros are excluded from the sample, a number that represents $7.55 \%$ of the 170,946 recorded transactions. This leads to a final sample of 4, 279 matches, out of which 1,733 have been recorded before the introduction of the ETF and 2, 546 afterwards.

Since we require that call and put transactions occur within a one-minute interval, we can only test the market efficiency at fixed points in time. The matching procedure might produce numerous pairing only when market activity is high and no result at all when it is low. One question that naturally arise is whether our sample of matchings is representative of the whole market activity. As it can be seen in the upper part of table 1 , almost $80 \%$ of the pairings have less than one month to maturity, and the number of pairing decrease with the time to maturity. This is consistent with the trading activity of both put and call options series on the MONEP that is highly concentrated on the nearby maturity. Figure 1 represent the intradaily activity of both put and call options transactions by classes of fifteen minutes. Trading activity slowly decrease from the market opening until $2.00 \mathrm{pm}$ and then rise throughout the afternoon to reach a maximum at the close. The distribution of pairings, represented on the same figure, follow the same intradaily trend. More matched pairs are obtained when the activity is higher, but still, a significant number of matches is obtained in the middle of the day, when the activity is at its lowest. Our sample of matching pairs are representative of the market activity in call and put options, and our results may offer a fair view of the trends to efficiency.

The bottom of table 1 reports the TTEs both for the full sample $(4,279$ put-call parity matches), and for the observations observed before (1,733 matches) and after (2,546 matches) the introduction of the ETF on the CAC 40 index. The 432 matches (10.10\% of the full sample) that don't exhibit a return to efficiency before the market close are gathered in sample A. The mean of the time to market close is of 106.27 minutes whereas its median equals 31.83 minutes. This sample is constituted by a low proportion of matches identified early in the trading day with prices remaining inefficient for a long period until the market close. (These observations are likely to represent highly inefficient options series that must not be dropped out when estimating the determinants of the time to efficiency. pas forcément le bon endroit pour dire ça) A higher proportion of the matches not returning to efficiency are observed within the last 30 minutes

\footnotetext{
${ }^{13}$ When the pairing is not synchroneous, we match it with the index value prevailing at the time of the second transaction. It is consistent with arbitrageurs that would monitor the options market and try to build the arbitrage portfolio only once an opportunity has been identified.
} 
of the trading day, the market having less time to adjust. Time to close dramatically decrease for the period following the introduction of the ETF. Hence, if their proportion remains of the order of $10 \%$ of the full sample, matches are much closer to the end of the trading day once the ETF is available. The market is not highly active for the corresponding series but transactions occurs and the new prices are not compatible with no arbitrage. The average number of call and put transactions occurring between the time of matching and the market close is 2.33 and 1.54, respectively. It diminishes with the introduction of the ETFs, like the time to close.

Sample B gathers the 3,847 matches returning to efficient prices before the end of the trading day. On average, one transaction is enough to nullify the arbitrage profit that nevertheless remain positive for 19.22 minutes. However, the distribution of TTEs, represented in figure ??, has a very fat left tail and is right skewed and the median TTE is of 3.06 minutes only. Spot trading of the index appears to increase market efficiency: TTE as well as the number of transactions needed to nullify the arbitrage profit are significantly lower once ETFs have been available on the CAC 40 index. Most of the opportunities disappear very quickly, apparently arbitraged. Still, it takes more than one hour for prices to be brought back to levels compatible with no arbitrage for $10.91 \%$ of the matches that exhibit a return to efficiency and the maximum TTE is of more than six hours whether it be before or after the introduction of the ETF.

On the whole, the market looks efficient but there appear to be a high degree of variability in the returns to efficiency. Some opportunities disappear very rapidly whether others remain for hours incompatible with no arbitrage. The next section is devoted to the analysis of the forces that drive the market prices back to efficient values.

\section{Empirical results}

This section is devoted to the empirical analysis of the determinants of the time-to-execution for the data described in the previous section using the survival approach described in section 2.3. In section 4.1, we detail the the explanatory variables we use and section 4.2 presents the parameter estimates. We check for the adequacy of our parametrization in section 4.3 and we discuss the implications of our estimates in section 4.4 .

\subsection{Explanatory variables}

To understand the reasons why some arbitrage opportunities disappear faster (if ever) than others, we use several explanatory variables which aim at capturing the trading environment that prevails at the time we match options trades that deviate from put-call parity. Arbitrageurs 
Table 1: Time to efficiency statistics

\begin{tabular}{|c|c|c|c|}
\hline & $\begin{array}{c}\text { Full } \\
\text { Sample }\end{array}$ & $\begin{array}{c}\text { Before } \\
\text { Introduction }\end{array}$ & $\begin{array}{c}\text { After } \\
\text { Introduction }\end{array}$ \\
\hline Observations & 4,279 & 1,733 & 2,546 \\
\hline \multicolumn{4}{|l|}{ by time to maturity } \\
\hline less than 1 month & 3,380 & 1,420 & 1,960 \\
\hline 2-3 months & 567 & 208 & 359 \\
\hline 4-6 months & 209 & 48 & 161 \\
\hline 7-12 months & 115 & 53 & 62 \\
\hline \multicolumn{4}{|c|}{ Sample A: no return to efficient prices before the market close } \\
\hline number & 432 & 178 & 254 \\
\hline proportion & 10.10 & 10.27 & 9.98 \\
\hline \multicolumn{4}{|l|}{ Transactions to close } \\
\hline calls & 2.33 & 3.11 & 1.78 \\
\hline puts & 1.54 & 2.51 & 0.86 \\
\hline \multicolumn{4}{|c|}{ Time to close (minutes) } \\
\hline mean & 106.27 & 147.17 & 77.06 \\
\hline median & 31.83 & 68.77 & 13.88 \\
\hline \multicolumn{4}{|c|}{ Sample B: return to efficient prices before the market close } \\
\hline number & 3,847 & 1,555 & 2,292 \\
\hline proportion & 89.90 & 89.73 & 90.02 \\
\hline \multicolumn{4}{|c|}{ Transactions to efficiency } \\
\hline calls & 0.90 & 1.10 & 0.77 \\
\hline puts & 0.89 & 1.23 & 0.66 \\
\hline \multicolumn{3}{|c|}{ Time to efficiency (minutes) } & 13.93 \\
\hline t-test & & \multicolumn{2}{|c|}{$7.91^{* * *}$} \\
\hline median & 3.06 & 4.90 & 3.32 \\
\hline Mann-Whitney test & & \multicolumn{2}{|c|}{$2,000,196^{* * *}$} \\
\hline
\end{tabular}


face liquidity risk -the risk of adverse price movements- which, following Kamara and Miller (1995), we proxy by liquidity measures for the options series and the underlying asset. First, we hypothesize that the more illiquid the options market, the harder for traders to establish arbitrage portfolios and the longer it will take for an arbitrage opportunity to disappear. Second, since arbitrageurs have to trade the underlying index, the same argument applies to index volatility. Third, ETF are available on CAC 40 index since January 21, 2001, easing spot trades in the index, which should reduce the time when inefficiencies persist.

Five out of our six explanatory variables are related to the prevailing conditions on the options market whereas the remaining two are related to the underlying asset. Variables 1-4 are designed to capture the liquidity of the options market along its various dimensions: ActivOpt and RatioOpt are proxies for the 'instantaneous' liquidity of the options series, whereas Maturity and MoneyClass are proxies for the 'intrinsic' liquidity of an option, irrespective of market conditions (trading concentrates on the nearby maturity and near-the-money options). Variables 5 and 6 are related to the risk and easiness of executing the index leg of the arbitrage. Volat measures the volatility of the underlying asset, and ETF identifies the pre- and post-introduction of the CAC 40 ETF periods since we assume that trading the index is easier when ETFs are available.

\subsubsection{Options Market}

1. ActivOpt $\equiv(\#$ calls $+\#$ puts $)$

is the total number of trades for the series of calls and puts which have to be included in the arbitrage portfolio, over the corresponding trading day.

2. RatioOpt $\equiv \mid \#$ calls $-\#$ puts $\mid$ /ActivOpt

measures the difficulty to execute the option leg due to existing imbalances in the activity of call and put series.

\section{Maturity}

is a categorical variable which takes value 1 for options that expire by the end of the current month, 2 for options whose maturity is comprised between 2 to 3 months, 3 for options whose maturity is comprised between 4 to 6 months and 4 otherwise.

\section{MoneyClass2, MoneyClass3, MoneyClass4}

are dummy variables which take value one if the option belongs to the corresponding moneyness class and zero otherwise. The moneyness classes are constructed in the fol- 
lowing way. We form four classes by computing the $15 \%, 50 \%$ and $85 \%$ quantiles from the empirical distribution of $S / X$, where $S$ denotes the index value and $X$ the options strike. We then assign the options belonging to the $15 \%$ lowest observed $S / K$ values (the deepest out-of-the-money calls and in-the-money puts) to class 1, whereas the $85 \%$ highest $S / K$ values (deepest in-the-money calls and out-of-the-money puts) are assigned to class 4 . Options belonging to classes 2-3 correspond to nearly-at-the-money calls and puts.

\subsubsection{Underlying Asset}

\section{Volat}

is the estimator of the index volatility expressed in an annual basis. We first compute a 10 -minute volatility from the 20 index values that immediately precede a given matching using the high-low Parkinson (1980) estimator

$$
\hat{\sigma}_{10}=\sqrt{\frac{\left(\ln P_{\max }-\ln P_{\min }\right)^{2}}{4 \ln 2}}
$$

where $P_{\max }$ and $P_{\min }$ are the maximum and the minimum of the index value over the 10-minute interval ${ }^{14}$. We then annualize the 10 -minute volatility using:

$$
\hat{\sigma}_{\text {year }}=\hat{\sigma}_{10} \sqrt{6 \times 8.5 \times 254}
$$

\section{ETF}

is a dummy variable which takes value 0 prior to the introduction of the CAC 40 exchange traded fund and 1 after.

\subsection{Parameter estimates}

This section presents the estimation results for the accelerated-failure-time specification of time to efficiency, using the maximum likelihood approach described in section 2.3.4. The estimated parameters, along with their corresponding standard errors and z-statistics are reported in table 2 .

\footnotetext{
${ }^{14}$ Notice that the Parkinson estimator requires regularly-spaced price series, which is the case here since the CAC 40 index is disseminated every 30 seconds.
} 
Table 2: Parameter estimates

This table reports the maximum likelihood parameter estimation for the 4.271 sample matchings using the accelerated-failure-time specification

$$
\begin{aligned}
\ln T= & \mu+\gamma_{1} \text { ActivOpt }+\gamma_{2} \text { RatioOpt }+\gamma_{3} \text { Maturity } \\
& +\gamma_{4} \text { MoneyClass } 2+\gamma_{5} \text { MoneyClass } 3+\gamma_{6} \text { MoneyClass } 4 \\
& +\gamma_{7} \text { Volat }+\gamma_{8} \text { ETF }+u
\end{aligned}
$$

where $u$ is an error term which follows a standard extreme value distribution.

\begin{tabular}{lrrr}
\hline \hline Parameter & Estimate & Std. error & z-statistic \\
\hline$\mu$ & 6.163 & 0.141 & 43.57 \\
$\gamma_{1}$ & -0.007 & 0.001 & -8.53 \\
$\gamma_{2}$ & 0.540 & 0.109 & 4.98 \\
$\gamma_{3}$ & 0.946 & 0.051 & 18.62 \\
$\gamma_{4}$ & 0.406 & 0.085 & 4.78 \\
$\gamma_{5}$ & 0.395 & 0.086 & 4.58 \\
$\gamma_{6}$ & 0.510 & 0.099 & 5.13 \\
$\gamma_{7}$ & -3.210 & 0.402 & -7.99 \\
$\gamma_{8}$ & -0.734 & 0.056 & -13.10 \\
$\ln \sigma$ & 0.508 & 0.012 & 42.98 \\
\hline \hline
\end{tabular}

The parameter estimates yield several interesting results we shall now discuss. We first restrict our attention to the general pattern of survival times before studying the effect of the explanatory variables.

Using (17) to transform the $\hat{\mu}$ and $\hat{\sigma}$ estimates back onto the original time scale gives the corresponding $\hat{\alpha}=0.022$ and $\hat{\lambda}=0.603$ values for the underlying Weibull distribution. From those results, it appears that the sample times-to-efficiency exhibit a decreasing hazard rate, so that the probability of an arbitrage opportunity disappearing in the next instant is higher shortly after it has been detected than after a long time period has elapsed. Probably, this (unconditional) pattern simply reflects differences across arbitrage opportunities according to the arbitrage profit they give rise to. Large profit opportunities may trigger immediate reaction from market participants thus resulting in a very short time to efficiency. On the contrary, small profit opportunities may be kind of neglected by traders as the total cost, combined with the associated risk and the difficulties they face when building the arbitrage portfolio, could result in a net loss.

Estimates associated with our explanatory variables somehow confirm this story while bring- 
ing additional interesting information. The coefficient on variable ActivOpt is negative with z-statistic -7.92 , indicating that the most active the options market, the shorter the time to efficiency. On the contrary, the coefficient on variable RatioOpt is significantly positive, which implies that the larger the trades imbalance between call and put options, the longer the time to efficiency. This is not surprising since difficulties to match, say a call option, with the corresponding put option will make things harder and longer for a given trader when she has to build the appropriate arbitrage portfolio. Overall, the results from the ActivOpt and RatioOpt variables suggest that the liquidity on the options market is a significant determinant for time to efficiency.

The coefficients on variables Maturity, MoneyClass2, MoneyClass3 and MoneyClass4 are all positive and significantly different from zero. The positive relationship we get between Maturity and TTE is not surprising since the nearest contracts are the most actively traded ones. By contrast, the coefficients on the moneyness categorical variables are more intriguing. In line with the liquidity argument, we would expect a negative coefficient on variables MoneyClass2 and MoneyClass3 since near at-the-money options are usually more frequently traded than other options. Remember however that from our definition of moneyness classes, options belonging to class 1 include both deeply out-of-the-money call options and deeply in-the-money put options, with the latter being rather actively traded by investors who seek to protect their portfolio against downward market movements. In this view, the positive coefficients associated to the moneyness classes 2 to 4 reflect a lower liquidity for these options compared with the liquidity of the deeply out-of-the-money put options market.

We now turn to the determinants in connection with the underlying index. We find evidence of a significant negative relationship between volatility and time to efficiency. This is ans interesting result since the direction of the effect was rather unpredictable. On the one hand, in line with Kamara and Miller (1995), volatility makes arbitrage riskier since it increases the probability for a trader to face adverse price changes by the time she is in the process of building the arbitrage portfolio. This should result in a longer time to efficiency. On the other hand, a mechanistic effect is at work by which a greater volatility increases the probability for the index price to be consistent with the Put-Call parity relationship in the next instant. Notice that this situation should occur more frequently in presence of 'small' arbitrage opportunities, but overall the mechanistic effect appear to be the dominant one in our sample.

Finally the negative coefficient on the ETF variable indicates that the introduction of the CAC 40 ETF by January 21, 2001 resulted in a shorter time to efficiency, and thus in an improved efficiency. This result may be compared with the findings of Ackert and Tian (1998, 2001) who find that the introduction of ETFs did not contributed to reduce ex post profits, nei- 
ther on the US market nor on the Canadian market. However, it is consistent with the evidence in Kurov and Lasser (2002) who document a negative relationship between the existence of the QQQ ETF on Nasdaq and the size of arbitrage profits on the corresponding futures contracts. However, our results provide a somewhat different information: by making trades on the underlying index easier, the ETF allows market participants to react in a shorter time interval once an arbitrage opportunity has been detected.

\subsection{Checking model adequacy}

To asses the appropriateness of our econometric specification, we performed a graphical (hazard plot) test of goodness-of-fit in the spirit of Lo, MacKinlay and Zhang (2002). Graphical tests are informal tests as "they serve as a means of rejecting clearly inappropriate models, not to 'prove' that a particular model is correct" (Klein and Moschberger, 2003). The underlying principle is to check whether the distributions of times-to-event, conditional on a set of covariates, follows the postulated distribution. In our case, this amounts to testing whether TTEs, conditional on our explanatory variables, follow a Weibull distribution.

To do so, we proceeded in the following way. We first computed the set of standardized residuals $\left\{\hat{u}_{i}\right\}$ as:

$$
\hat{u}_{i}=\frac{\ln T_{i}-\hat{\mu}-\gamma^{\prime} Z_{i}}{\hat{\sigma}}
$$

If the Weibull specification holds then, according to (20), the $\left\{\hat{u}_{i}\right\}$ should behave like a censored sample from a standard extreme value distribution. To check this point, we computed the Kaplan-Meier ${ }^{15}$ estimator $\hat{S}\left(\left\{\hat{u}_{i}\right\}\right)$ of the survival curve from the $\left\{\hat{u}_{i}\right\}$. Taking minus the log, this yields the integrated hazard $\hat{\Lambda}\left(\left\{\hat{u}_{i}\right\}\right)$. Using (21), the expression for the integrated hazard of a standard extreme value distribution is found to be:

$$
\Lambda_{U}(u)=\exp (u)
$$

so that, if our Weibull specification holds, a plot of $\left\{\hat{u}_{i}\right\}$ against $\left\{\ln \left[\hat{\Lambda}\left(\hat{u}_{i}\right)\right]\right\}$ should be a straight line with intercept 0 and slope 1 . The result from the plot is reported in figure 3. Although we observe some departures from the expected straight line, these are sufficiently small to consider that our model reasonably fit the data ${ }^{16}$.

\footnotetext{
${ }^{15}$ The Kaplan-Meier estimator is a non-parametric technique which allows to compute the empirical survival function from a sample including censored observations. Interested readers may refer to the original article, Kaplan-Meier (1958), or to Lo, MacKinlay and Zhang (2002).

${ }^{16} \mathrm{We}$ also performed hazard plot tests using other specifications for the underlying distribution of times, namely exponential, log-logistic and log-normal. The hazard plots we obtained clearly reject the exponential specification,
} 


\subsection{Implications for time to efficiency}

Having tested the appropriateness of our specification and with parameter estimates in hands, we are now interested in computing the implications of our model for the survival of arbitrage opportunities through a sensitivity analysis. In order to study to which extent initial market conditions $\left(Z_{i}\right)$ impact the time to efficiency, we computed the corresponding survival function $S_{W}\left[t \exp \left(\gamma^{\prime} Z_{i}\right)\right]$, using the Weibull specification (see eq. 12) for the baseline survival function $S_{W}$. The analysis is performed for each of the determinants we identified by allowing the explanatory variable of interest to take its $10 \%, 25 \%, 50 \%, 75 \%$ and $90 \%$ percentile values while all other explanatory variables are held fixed at their sample median value ${ }^{17}$. The results are reported in figure 4.

Figure 4 shows, as expected, that the higher the volatility, the higher is the probability the arbitrage opportunity has disappeared over any given time interval. However, the differences in survival curves are not so important. If we consider for example the situation that prevails 20 minutes after an arbitrage opportunity has been detected, the probability for the market to remain in a state a profitable arbitrage is $34.10 \%$ for the highest $10 \%$ volatilities versus $44.98 \%$ for the lowest $10 \%$. This conclusion remains valid for the ActivOpt and the RatioOpt variables. If we refer to the former, it appears that the survival probability after a 20 -minute interval is $46.78 \%$ for the highest $10 \%$ ActivOpt values versus $33.48 \%$ for the lowest $10 \%$. Regarding the RatioOpt variable, the difference is only $8.67 \%$ between the top and the bottom decile $(46.88 \%$ versus $38.21 \%$ respectively).

Variables referring to maturity, moneyness and the existence of the ETF yield more distinct patterns across the different values we use for the underlying determinant. The most dramatic changes occur with respect to the maturity class. For the longest maturities, the probability an arbitrage opportunity has survived after 20 minutes is $75.60 \%$, with a corresponding $21.36 \%$ probability for the shortest maturities. It takes only 5:28 minutes for half of the opportunities to disappear for the shortest maturities whereas $88.20 \%$ of them are still alive at the same time if the underlying options is to expire after 6 months. The same conclusion apply to the categorical variables associated with the moneyness classes. $44 \%$ of the moneyness class 4 arbitrage opportunities are still alive 20 minutes, but this figure drops to $32.77 \%$ for the moneyness class 1 options. Moreover, there are only slight differences across options belonging to the last 3 mon-

whereas the other two distribution yield clearly inferior, though acceptable fit compared with the Weibull specification. We also tried to fit the data using a generalized gamma specification, but were unable to get converging result when performing the likelihood maximization. All results are available upon request.

${ }^{17}$ When fixed, dummy and categorical variables are assigned the following values: $\mathrm{ETF}=1$, Maturity $=2$, MoneyClass2 = 1, MoneyClass3 = 0, MoneyClass $4=0$. 
eyness classes. Overall, these results stress the importance of the intrinsic liquidity and options characteristics for the profitability of arbitrage-based strategies and the efficiency of the options market. Also, an implication of these results is that arbitrageurs tend to concentrate their actions on the options which, by their very nature, are likely to be the most traded ones.

We finally turn to the ETF variable. As stated before, the existence of an ETF on the underlying CAC index resulted in a shorter time to efficiency. More precisely, results associated with the survival curses show that, by 20 minutes, $58.25 \%$ of arbitrage opportunities were still alive prior the ETF was introduced, a figure that drops to $42.97 \%$ after the introduction.

\section{Conclusion}

Using the concept of TTE, this article sheds light on the determinants which govern the speed at which an index options market converges to a state of efficiency after an arbitrage opportunity has been detected. To achieve this goal, we make use of a statistical technique called survival analysis. We find that the Weibull distribution along with an accelerated failure time specification provides a sensible fit to the data and that the TTE is quite sensitive to several explanatory variables. We find that the activity on the options markets as well as the possibility to easily match a given call with the corresponding put significantly influence the TTE. We also document that differences in options characteristics (namely moneyness and distance to maturity) lead to important changes in the probability for an arbitrage opportunity to survive over a given time-interval. Regarding the underlying asset, we find that survival times are shorter when the index volatility is high. Finally, the introduction of an ETF replicating the underlying index results in shorter TTEs, underlying the positive effect this new instrument has on index derivatives markets efficiency. 


\section{References}

ACKERT, L. F., AND Y. S. TIAN (1998): “The Introduction of Toronto Index Participation Units and Arbitrage Opportunities in the Toronto 35 Index Option Market," Journal of Derivatives, 5(4), 44-53.

(2001): "Efficiency in Index Options Markets and Trading in Stock Baskets," Journal of Banking and Finance, 25(9), 1607-1634.

Bharadwaj, A., And J. B. Wiggins (2001): "Box Spread and Put-Call Parity Tests for the S\&P 500 Index LEAPS Market," Journal of Derivatives, 8(4), 62-71.

Billingsley, R. S., And D. M. Chance (1985): "Options Markets Efficiency and the Box Spread Strategy," Financial Review, 20(4), 287-301.

Capelle-Blancard, G., and M. Chaudhury (2001): "Efficiency Tests of the French Index (CAC 40) Options Market,” Working paper, McGill University.

Cassese, G., And M. Guidolin (2001): "Pricing and Informational Efficiency of the MIB30 Index Options Market," Working paper, University of Southern Switzerland, http://www.lu.unisi.ch/istfin/papers/gianluca.pdf.

Cavallo, L., And P. Mammola (2000): "Empirical Tests of Efficiency of the Italian Index Options Market," Journal of Empirical Finance, 7, 173-193.

Chance, D. M. (1987): "Parity Tests of Index Options," Advances in Futures and Options Research, 2, 47-64.

Chesney, M., R. Gibson, And H. Loubergé (1995): “Arbitrage Trading and Index Option Pricing at SOFFEX: An Empirical Study Using Daily and Intradaily Data," Finanzmarkt und Portfolio Management, 9(1), 35-60.

Chordia, T., R. Roll, and A. Subrahmanyam (2004): "Evidence on the speed of convergence to market efficiency," Forthcoming in the Journal of Financial Economics.

Deville, L. (2004): "Time to Efficiency on Options Markets and the Introduction of ETFs: Evidence from the French CAC 40 Index," Working Paper, Paris Dauphine University.

Evnine, J., And A. Rudd (1985): "Index Options: The Early Evidence," Journal of Finance, 40(3), 743-756.

FinuCAne, T. J. (1991): "Put-Call Parity and Expected Returns," Journal of Financial and Quantitative Analysis, 26(4), 445-457.

Gould, J., AND D. Galai (1974): "Transaction Costs and the Relationship between Put and Call Prices," Journal of Financial Economics, 1, 105-129.

Greene, W. H. (2002): Econometric Analysis. Prentice Hall, Englewood Cliffs, NJ, 4 edn. 
Kamara, A., And T. W. Miller (1995): "Daily and Intradaily Tests of Put-Call Parity," Journal of Financial and Quantitative Analysis, 30(4), 519-539.

KAPlan, E. L., AND P. MEIER (1958): "Nonparametric Estimation from Incomplete Observations," Journal of the American Statistical Association, 53, 457-481.

KIEFER, N. (1988): "Economic Duration Data and Hazard Functions," Journal of Economic Litterature, 26, 646-679.

Klein, J., And M. Moeschberger (2003): Survival analysis: techniques for censored and truncated data, Statistics for biology and health. Springer, 2nd edn.

Klemkosky, R. C., And B. G. Resnick (1979): "Put-Call Parity and Market Efficiency," Journal of Finance, 34(5), 1141-1155.

Kurov, A. A., AND D. J. LASSER (2002): “The Effect of the Introduction of Cubes on the Nasdaq-100 Index Spot-Futures Pricing Relationship," Journal of Futures Markets, 22(3), 197-218.

Lo, A., C. MACKInlay, AND J. ZhANG (2002): "Econometric models of limit-order executions," Journal of Financial Economics, 65, 31-71.

Merton, R. C. (1973): “The Relationship between Put and Call Prices: Comment,” Journal of Finance, 28(1), 183-184.

Mittnik, S., And S. Rieken (2000): "Put-Call Parity and the Informational Efficiency of the German DAX-Index Options Market," International Review of Financial Analysis, 9, 259-279.

PARkinson, M. (1980): “The Extreme Value Method for Estimating the Variance of the Rate of Return," Journal of Business, 53, 61-65.

Puttonen, V. (1993): "Boundary Conditions for Index Options: Evidence from the Finnish Market," Journal of Futures Markets, 13(5), 545-562.

Stoll, H. R. (1969): “The Relationship between Put and Call Option Prices," Journal of Finance, 24, 801-822.

Wagner, D., D. M. Ellis, And D. A. Dubofsky (1996): "The Factors Behind Put-Call Parity Violations of S\&P 100 Index Options," Financial Review, 31(3), 535-552. 


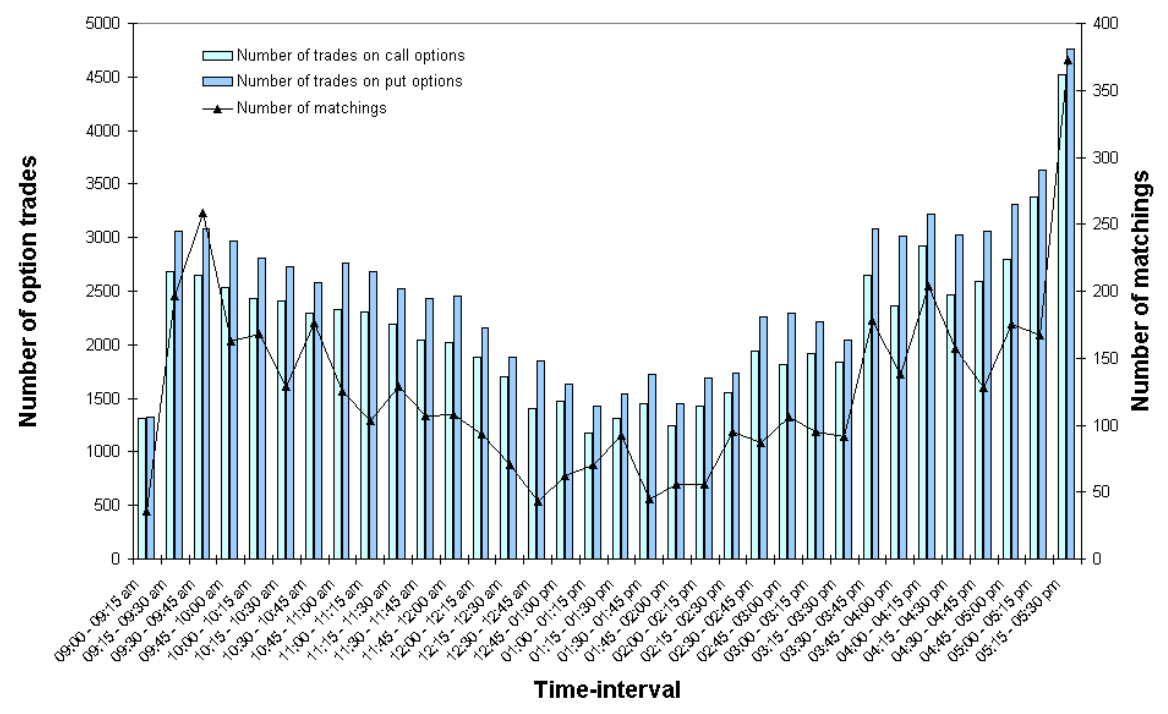

Figure 1: Daily repartition of call and put trades and put-call parity synchronous pairings

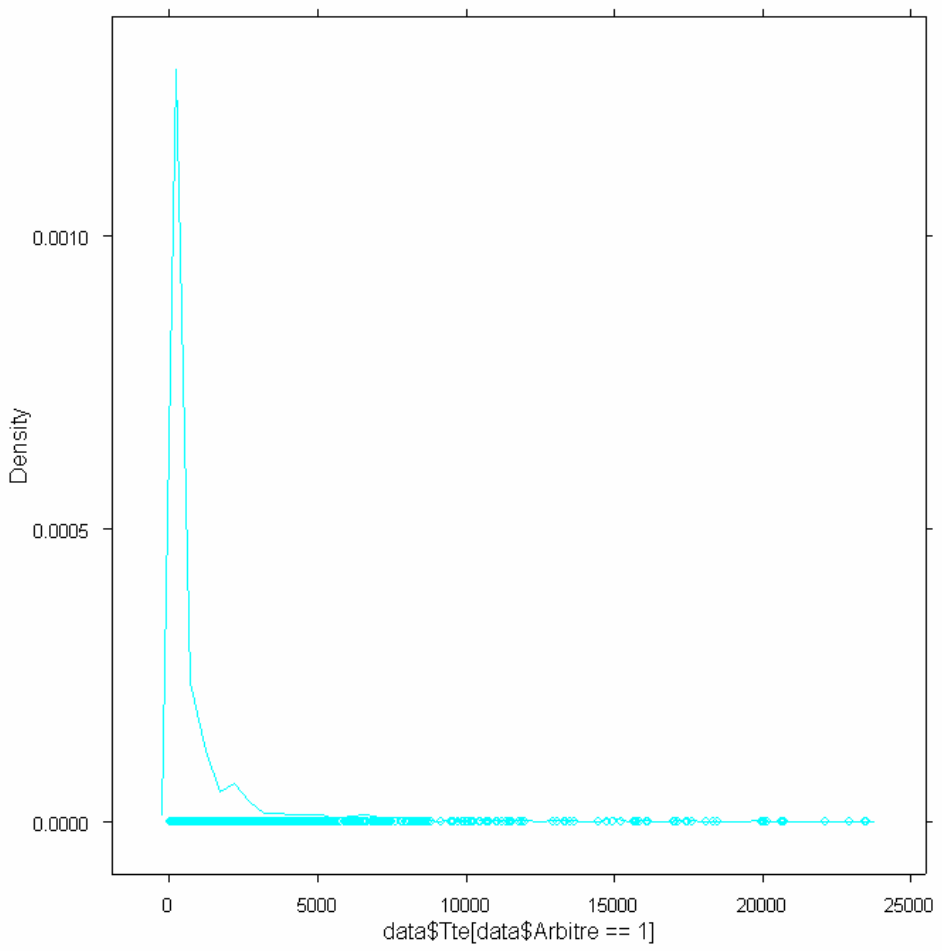

Figure 2: Censored empirical density of TTEs (3,847 observations returning to efficiency before the market close, $\mathrm{x}$-axis in seconds) 
Figure 3: Test of Goodness-of-fit

The graph plots the log of the integrated hazard of the standardized residuals based on the Kaplan-Meier estimator of their empirical survival curve $(\ln [\hat{\Lambda}(\hat{u})])$, against the standardized residuals $(\hat{u})$. Standardized residuals are computed using (23). Under appropriate specification for the model, the graph should coincide with the plotted straight line.

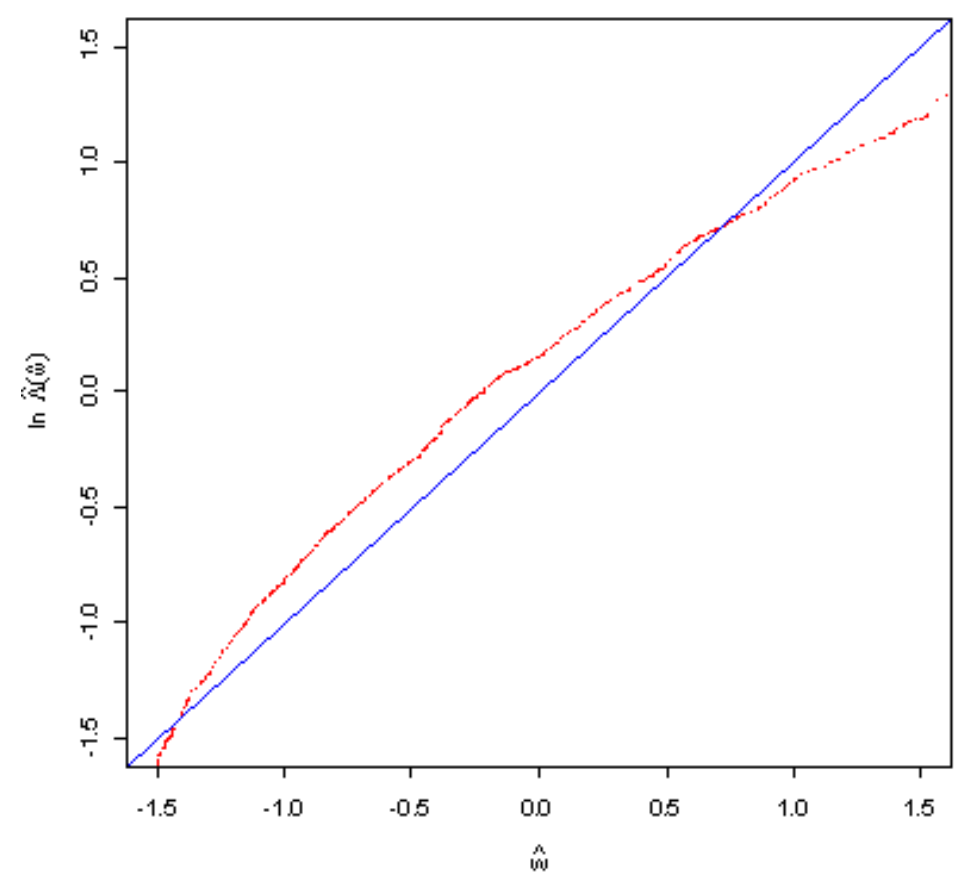


Figure 4: Sensitivity of survival functions to market conditions
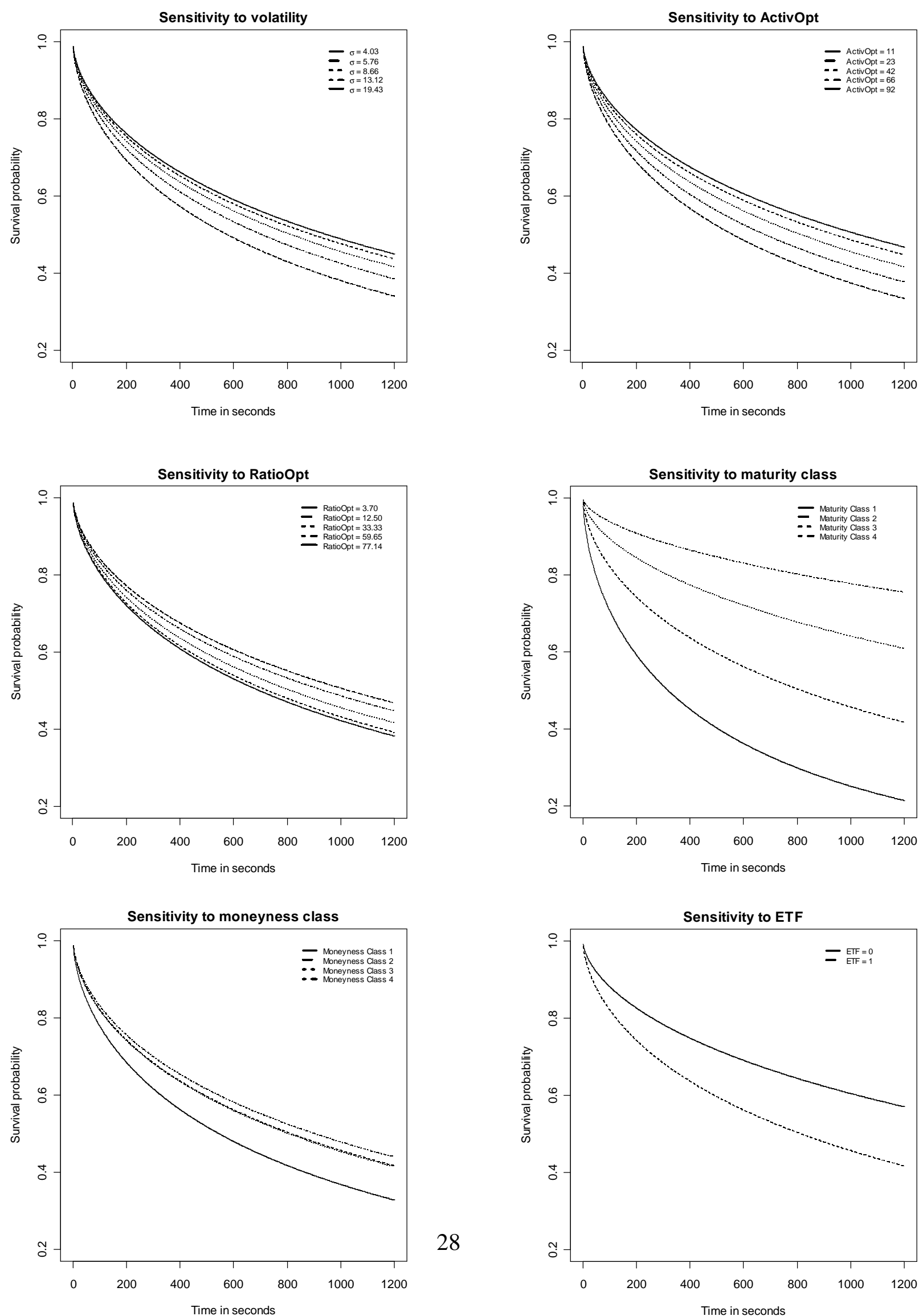